\title{
Computational insight into the capacitive performance of graphene edge planes
}

\author{
Cheng Zhan, ${ }^{\dagger}$ Yu Zhang, ${ }^{\dagger}$ Peter T. Cummings, ${ }^{\dagger}$ and De-en Jiang ${ }^{\dagger, *}$ \\ ${ }^{*}$ Department of Chemistry, University of California, Riverside, CA, 92521, United States \\ ${ }^{\dagger}$ Department of Chemical and Biomolecular Engineering, Vanderbilt University, Nashville, TN, \\ 37235, United States
}

*To whom correspondence should be addressed. E-mail: djiang@ucr.edu. Tel.: +1-951-8274430

Abstract: Recent experiments have shown that electric double-layer capacitors utilizing electrodes consisting of graphene edge plane exhibit higher capacitance than graphene basal plane electrodes. However, theoretical understanding of this capacitance enhancement is still limited. Here we applied a self-consistent electronic calculation on the electrode/electrolyte interface and found that the capacitance of graphene edge plane depends on the edge type: zigzag edge has higher capacitance than armchair edge. We further examined the quantum, dielectric, and electric double-layer (EDL) contributions to the total capacitance of the edge-plane electrodes. Through classical molecular dynamics simulation, we found that the edge planes have higher electric double-layer (EDL) capacitance than the basal plane due to better adsorption of counter-ions and higher solvent accessible surface area, indicating the importance of surface morphology in affecting EDL capacitance. Our work therefore has elucidated the capacitive energy storage in graphene edge planes that take into account both the electrode's electronic structure and the EDL structure.

Key Words: Graphene edge plane, capacitance, joint density functional theory, electric doublelayer, classical molecular dynamics 


\section{Introduction}

Capacitors are widely used electronic devices that store and release electric energy quickly. There are two types of capacitors: a dielectric capacitor achieves its energy storage through a dielectric medium [1-3] (such as an oxide layer); an electrochemical capacitor (also called “supercapacitor") stores electric energy based on surface redox reaction (“pseudocapacitor") or electric double layer ("electric double-layer capacitor" or EDLC). Compared with dielectric capacitors, supercapacitors have much higher energy density; compared with batteries, they have much higher power densities and long cycle lives [4-7]. Carbon materials, including activated carbons, graphene, and advanced porous carbons, are used as electrode materials for EDLCs because of their low cost, high conductivity, good chemical stability, and low density [8-15]. However, the capacitance of graphene is limited by its low electronic density of states (DOS) at the Fermi level [16-19]. This quantum capacitance can be separately computed through electronic density functional theory (DFT), while the EDL capacitance can be obtained through classical methods (molecular dynamics, classical DFT, or Monte Carlo) [18, 20-24].

To increase the quantum capacitance $\left(\mathrm{C}_{\mathrm{Q}}\right)$ and hence the total capacitance, one effective way is to increase the electronic density of states at the Fermi level for the electrode material via nitrogen functionalization and doping of the graphene electrode $[25,26]$ or using the lighter and more metallic borophene [27]. Several simulations have focused on how to improve $\mathrm{C}_{\mathrm{Q}}$ of graphene-based electrode [28-33]. For few-layer-graphene electrodes, our recent work showed that dielectric screening also contributes to the total capacitance [34]. Most of the theoretical studies on graphene systems have focused on the graphene basal plane $[13,18,28,31,33,35$, 36], while little has been done on the capacitive performance of graphene edge planes. Recent experiments have indicated that the graphene edge plane could have higher capacitance than graphene basal plane [37-41]. Theoretical understanding on the capacitance of graphene edge plane is still limited [42], and the dielectric contribution has not been taken into account to provide a complete physical picture of the capacitance behavior.

In this work, we apply the self-consistent joint density functional theory to simulate the graphene edge plane in contact with the electrolyte via an implicit solvation model. We will separate the contributions from quantum, EDL, and dielectric capacitances. To include explicit 
ion solvation, we will also apply classical molecular dynamics to investigate the contribution of the EDL capacitance of graphene edges.

\section{Methods}

\subsection{Joint density functional theory}

Electronic structure of the solvated electrode in implicit electrolyte is solved by joint density functional theory, using the JDFTx package with the charge-asymmetric nonlocally-determined local-electric (CANDLE) solvation model solvation $[43,44]$. The interface model is described by periodic boundary conditions: pristine graphite crystal is modeled in a $2.460 \times 2.460 \AA^{2}$ rhombus lattice in $\mathrm{ABAB}$ stacking pattern with the interlayer distance of $3.40 \AA$. We used the (110) and (100) surfaces of graphite to model the armchair edge and zigzag edge, respectively.

The $\mathrm{sp}^{2}$ carbon atom at the edge site is terminated by $\mathrm{H}$ atom with the $\mathrm{C}-\mathrm{H}$ bond length of 1.140 $\AA$. The vacuum layer thickness is $20 \AA$ and will be filled by implicit solvation model (6M monovalent aqueous electrolyte). The model is shown in Fig. 1. The exchange-correlation energy for the electronic system is described by Generalized-Gradient-Approximation with PerdewBurke-Ernzerhof functional (GGA-PBE) [45]. The ultrasoft pseudopotential is used to describe the nuclei-electron interaction for carbon and hydrogen atoms [46]. To accurately capture the electronic chemical potential shift, the kinetic energy cutoff of plane wave basis set is 30 hartree and the k-point mesh for Brillouin zone sampling is $24 \times 36 \times 1$ for armchair edge and $36 \times 24 \times 1$ for zigzag edge.

The total differential capacitance $\mathrm{C}_{\mathrm{D} \text {,tot }}$ is defined by: $C_{D, t o t}=e_{0} d Q / d \mu_{e}$, where $\mathrm{Q}$ is areanormalized excess charge density of electrode, $e_{0}$ is the elementary charge, and $\boldsymbol{\mu}_{\mathrm{e}}$ is the electronic chemical potential of the solvated electrode [34]. In this work, we change Q on the electrode and then obtain the corresponding shift in the electrode potential $\boldsymbol{\mu}_{\mathrm{e} /} e_{0}$, which can be understood as the applied potential or voltage. The integral capacitance $\mathrm{C}_{\text {tot }}$ is defined by: $C_{t o t}=e_{0} \Delta Q / \Delta \mu_{e}$. The shift in the electronic chemical potential $\left(\Delta \mu_{e}\right)$ includes three contributions:

$$
\begin{aligned}
& C_{\text {tot }}=\frac{\Delta Q}{\Delta \mu_{e} / e_{0}}=\frac{\Delta Q}{\varphi_{Q}+\varphi_{N Q}}=\frac{\Delta Q}{\varphi_{Q}+\varphi_{E D L}+\varphi_{\text {Dielec }}} \\
& C_{\text {tot }}^{-1}=C_{Q}^{-1}+C_{N Q}^{-1}=C_{Q}^{-1}+C_{E D L}^{-1}+C_{\text {Dielec }}^{-1}
\end{aligned}
$$


where $\mathrm{C}_{\mathrm{Q}}$ and $\mathrm{C}_{\mathrm{NQ}}$ correspond to quantum capacitance and non-quantum capacitance, respectively. $\mathrm{C}_{\mathrm{NQ}}$ originates from the electrostatic energy change of the electrode/electrolyte system due to the external electric field of EDL, which can be obtained from the band shift in the JDFT calculation [34]. $\mathrm{C}_{\mathrm{NQ}}$ is the total consequence of $\mathrm{C}_{\mathrm{EDL}}$ and $\mathrm{C}_{\text {Dielec }}$, which correspond to the EDL response of the electrolyte and the dielectric screening of the electrode, respectively. The EDL and dielectric screening contribution can be separated by plotting the electrostatic potential for an ideal planar electrode surface, such as Pt and graphene. However, these two contributions are not separable in the graphene edge plane electrode due to the difficulty in defining the electrolyte/electrode interface for such a non-ideal and corrugated surface. Thus, we will use classical molecular dynamics to better understand the surface morphology effect on the EDL capacitance.

(a)

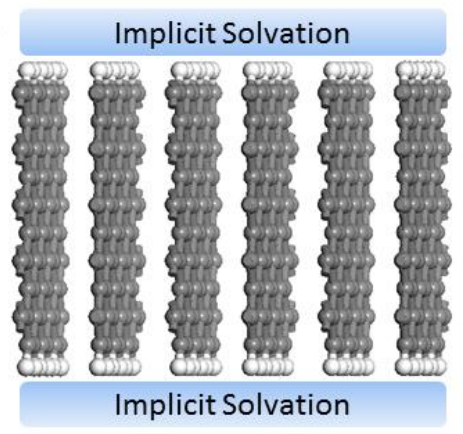

(c)

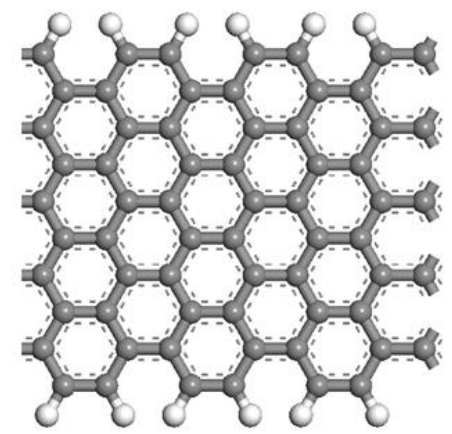

(b)

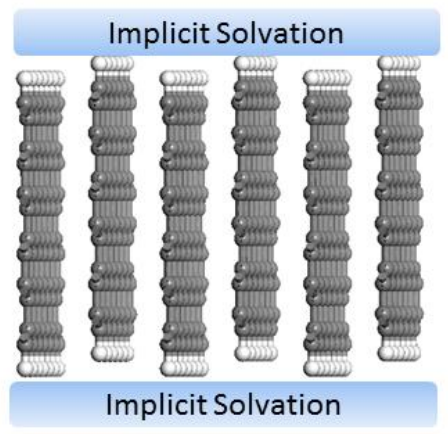

(d)

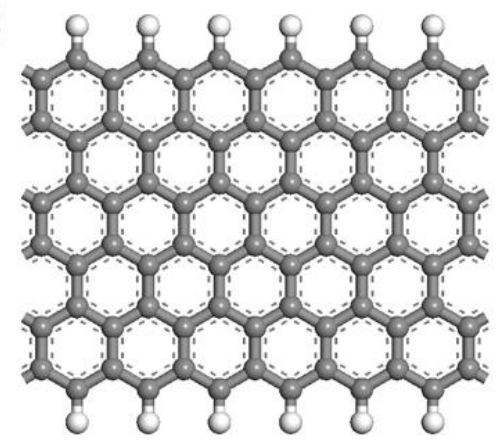

Fig. 1. The model used in the joint density functional theory calculation: (a) and (b) are the side view of graphite (110)-armchair and (100)-zigzag surfaces, respectively. (c) and (d) are the front view of single layer armchair edge and zigzag edge, respectively. 


\subsection{Classical molecular dynamics (CMD) simulations}

We applied CMD in the NVT ensemble using the GROMACS [47] package to simulate the EDL capacitance of different graphene edge planes. As illustrated in Fig. S1 in the supplementary data, each simulated system consists of a slab of $6 \mathrm{M} \mathrm{NaCl}$ aqueous solution enclosed between two electrodes, which were set at least $5.0 \mathrm{~nm}$ away to guarantee a bulk-like behavior of the electrolyte in the channel center. All the electrode atoms were fixed during the simulation. The force field parameters for the electrode and the electrolyte are adopted from our previous work [26]. When simulating the charged electrode, additional partial charges were added to surface $\mathrm{H}$ atoms only, since our DFT calculation showed that the excess charge mainly distributes on the $\mathrm{H}$ atoms at the interface (Fig. S2). The slab-PME method [48] was used to compute the electrostatic interaction, and the dimension vertical to the electrode surface was set to be 5 times the separation distance of the electrodes, to ensure that the accuracy of the electrostatic calculation is comparable to that of the two-dimensional Ewald method [49]. Each simulation was initiated at $800 \mathrm{~K}$ for $2 \mathrm{~ns}$, followed by $8 \mathrm{~ns}$ of annealing to $298 \mathrm{~K}$ and then equilibrated at $298 \mathrm{~K}$ for $10 \mathrm{~ns}$. Another production run of $10 \mathrm{~ns}$ was performed for data analysis. The temperature was controlled by using a Berendsen thermostat [50]. The time step of $1 \mathrm{fs}$ was applied. Each simulation was repeated three times with different initial configurations to reduce statistical errors. Of note, the constant-potential MD method can avoid the arbitrariness in assigning charges to the surface atoms and describe the EDL more accurately [51] than the constant-charge method used here. We chose the latter for its faster speed and simpler implementation, yet it still can capture the influence of surface morphology on the EDL capacitance and estimate the EDL contribution in total capacitance.

\section{Results and discussion}

\subsection{Electronic chemical potential $\left(\mu_{\mathrm{e}}\right)$ shift and potential at the point of zero charge $\left(\boldsymbol{\psi}_{\mathrm{PZC}}\right)$ for the graphene edge plane}

To determine the charge vs potential curve for the graphene edge-plane electrodes, we need to determine their $\boldsymbol{\psi}_{\text {PZC }}$. We calculated the electronic chemical potential $\left(\boldsymbol{\mu}_{\mathrm{e}}\right)$ of the graphene edge solvated in the implicit electrolyte with various electrode thicknesses. The calibrated Standard Hydrogen Electrode (SHE) in the CANDLE solvation model in JDFTx is -4.66 eV [44]. The calculated $\boldsymbol{\mu}_{\mathrm{e}}$ of the graphene basal plane at zero charge is $-4.0 \mathrm{eV}$, so its theoretical $\boldsymbol{\psi}_{\text {PZC }}$ is 
-0.6 V vs SHE. The experimental $\boldsymbol{\psi}_{\text {PZC }}$ of graphene is located at $-0.2 \mathrm{~V}$ to $0.1 \mathrm{~V}$ vs SHE [52]. Table 1 shows the calculated $\boldsymbol{\mu}_{\mathrm{e}}$ of neutral graphene edges (both armchair and zigzag type) of different thickness (see Fig. S3 and S4 for the range of thickness). Consistent with a previous theoretical study on the work function of graphene nanoribbon that the graphene edge has lower work function than pristine graphene [53], we found that $\boldsymbol{\mu}_{\mathrm{e}}$ of neutral graphene edges are higher than that of the graphene basal plane. The theoretical $\boldsymbol{\psi}_{\mathrm{PZC}}$ of a graphene zigzag edge is $-1.6 \mathrm{~V}$ vs SHE, while that of the graphene armchair edge is $-1.7 \mathrm{~V}$ vs SHE, so the graphene edge planes are more suitable to be the anode material for positive charge. In addition, Table 1 shows that the thickness of edge plane has minor influence on $\boldsymbol{\psi}_{\text {PZC }}$, a trend also found in few-layer graphene systems [13].

Table 1

Electronic chemical potential $\left(\boldsymbol{\mu}_{\mathrm{e}}\right)$ of armchair and zigzag graphene edges of different thickness at zero surface charge and the corresponding potential $\left(\boldsymbol{\psi}_{\mathrm{PZC}}\right)$ at the point of zero charge vs standard hydrogen electrode (SHE). (see Fig. S3 and S4 for the so-named edge structures).

\begin{tabular}{ccc}
\hline Edge Structure & $\boldsymbol{\mu}_{\mathrm{e}}(\mathrm{eV})$ & $\boldsymbol{\psi}_{\text {PZC }}$ Vs SHE $(\mathrm{V})$ \\
\hline Armchair-1 & -2.93 & -1.73 \\
Armchair-2 & -2.84 & -1.81 \\
Armchair-3 & -2.95 & -1.71 \\
Armchair-4 & -2.93 & -1.72 \\
Armchair-5 & -2.87 & -1.78 \\
Zigzag-1 & -3.06 & -1.59 \\
Zigzag-2 & -3.09 & -1.57 \\
Zigzag-3 & -3.09 & -1.56 \\
Zigzag-4 & -3.09 & -1.57 \\
Zigzag-5 & -3.09 & -1.57 \\
\hline
\end{tabular}

\subsection{Charge-potential curve of graphene edges in contact with an implicit solvation model}

The calculated electrode charge vs electrode potential curve is plotted in Fig. 2a and 2b for the armchair and zigzag edges, respectively; the differential capacitance is plotted in Fig. $2 \mathrm{c}$ and 
2d, obtained by applying numerical differentiation on the fitted charge-potential curves. In Fig. $2 \mathrm{a}$ and $2 \mathrm{c}$, one can see that the capacitance of the armchair edge shows strong dependence on the potential near PZC and then becomes flat at high voltage. This is due to the semiconducting nature of the armchair edges: the Fermi level is located in the middle of the band gap (the electronic density of states, DOS, is shown in Fig. S5), which causes a greater $\boldsymbol{\mu}_{\mathrm{e}}$ shift when the edge plane is charged from its neutral state. At higher voltage $(>1 \mathrm{~V})$, the differential capacitance of the armchair edge loses its strong voltage dependence due to a quite flat DOS below the band gap, as shown in Fig. S5. Armchair-3 edge has higher capacitance at PZC than other edges (Fig. 2c) due to its narrower gap and special electronic structure (see Fig. S5). Unlike armchair edges, zigzag edges have higher capacitance which is less voltage dependent (Fig. 2d). This can be explained by the special edge state of the zigzag edges (see Fig. S6), which yields a large DOS at the Fermi level and makes the quantum capacitance contribution $\left(1 / \mathrm{C}_{\mathrm{Q}}\right)$ trivial.

(a)

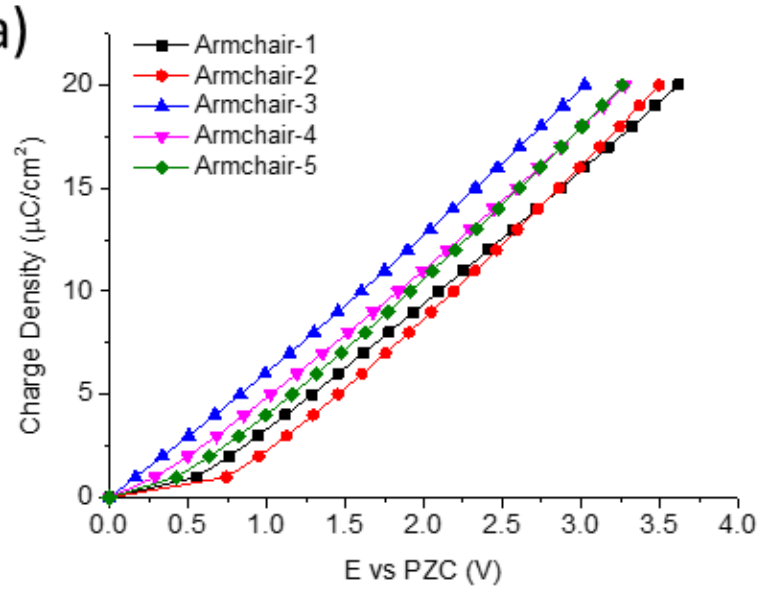

(c)

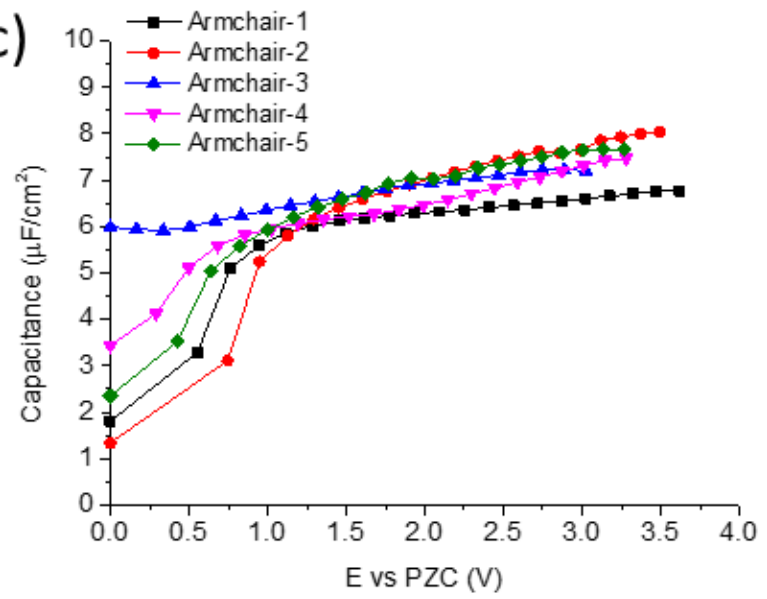

(b)

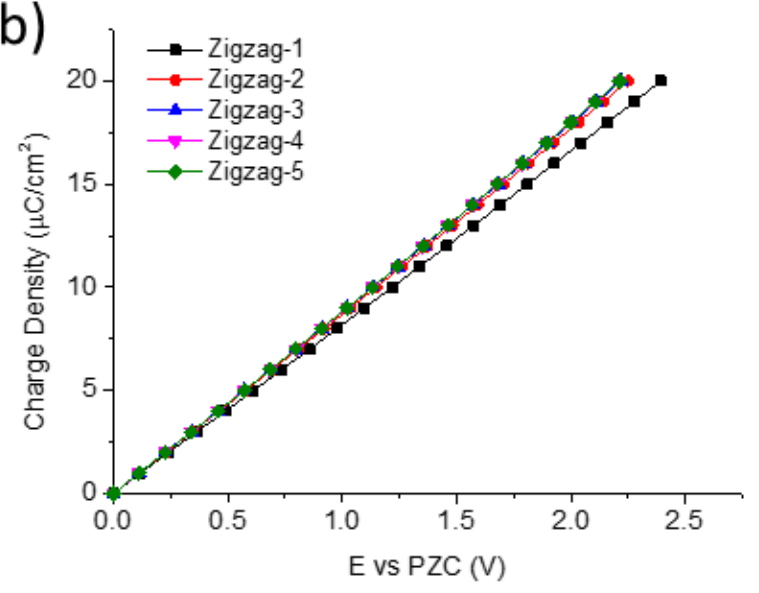

(d)

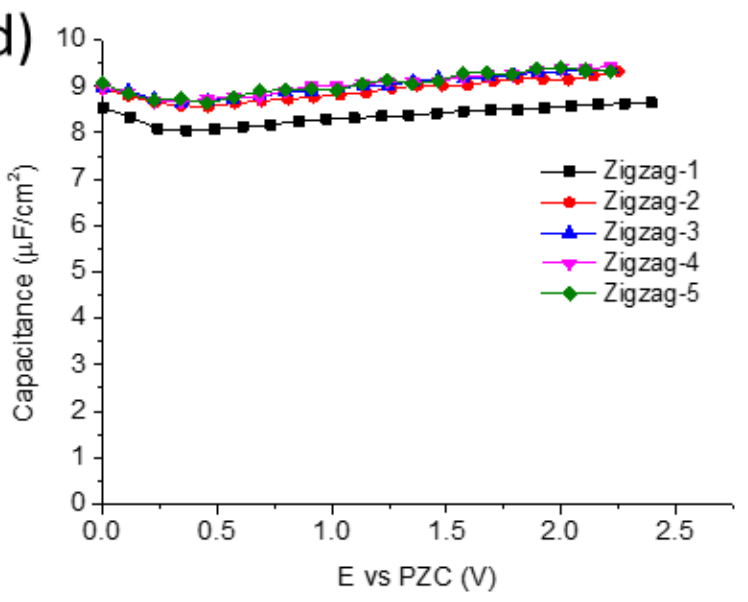


Fig. 2. Charge vs potential (a, b) and differential capacitance (c, d) vs potential curves for the armchair edge $(a, c)$ and the zigzag edge $(b, d)$ of different electrode thickness. Armchair 1

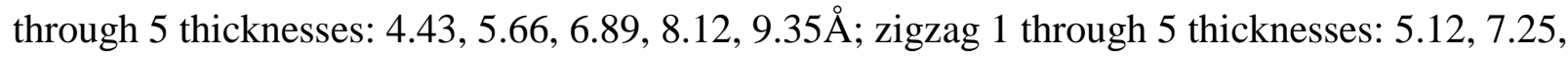
9.38, 11.51, $13.64 \AA$ A. Corresponding structures are in Fig. S3 and S4.

(a)

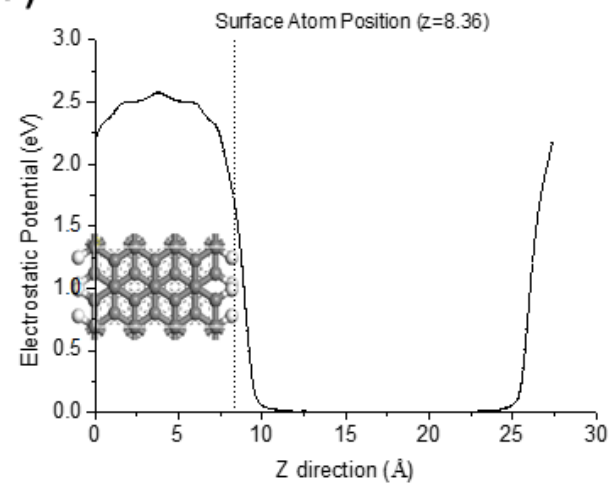

(c)

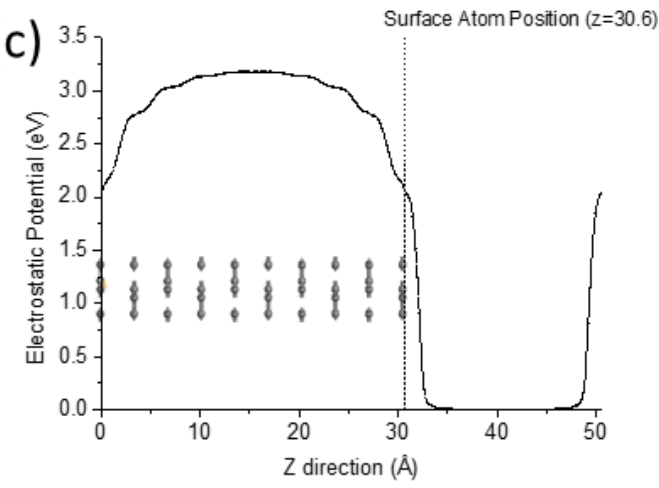

(b)

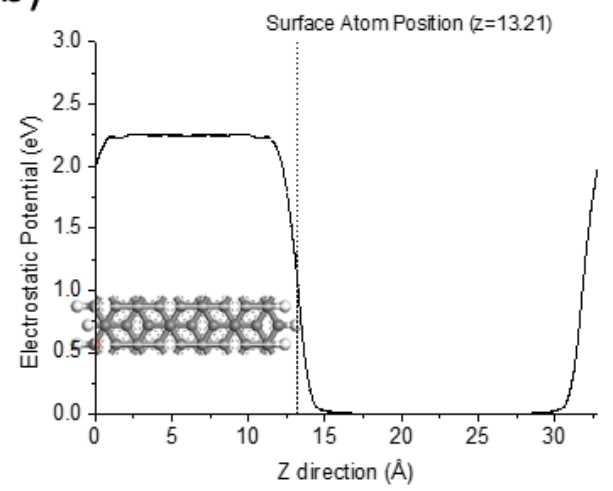

(d)

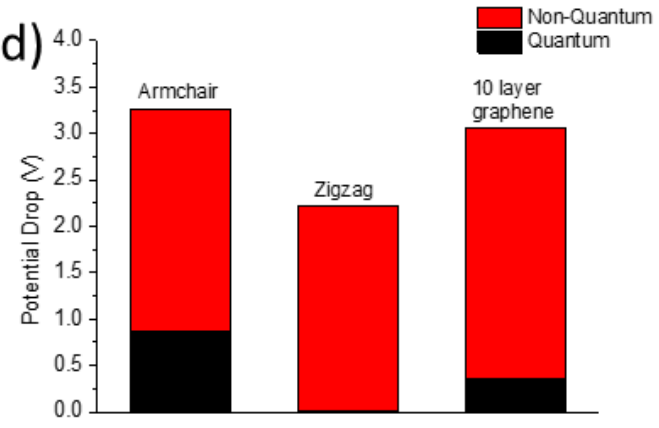

Fig. 3. Planar-average electrostatic potential drop in the electrode, $\Delta \boldsymbol{\psi}(\mathrm{r})$, along the electrode surface normal at a fixed surface charge density of $20 \mu \mathrm{C} / \mathrm{cm}^{2}$ for: (a) armchair edge, (b) zigzag edge, and (c) 10-layer graphene; (d) is the corresponding total potential drop including both quantum (Fermi level shift) and non-quantum (band shift) contributions. The corresponding planar average charge distribution is plotted in Fig. S2. The dashed lines in (a), (b) and (c) indicate the position of surface atoms that could be roughly treated as the electrode/electrolyte interface. 


\subsection{Electrostatic potential drop inside the electrode}

To understand the charging inside the graphene edge plane, we plotted the planar-average total electrostatic drop, $\Delta \boldsymbol{\psi}(\mathrm{r})$, of a positively charged edge plane in Fig. 3. As shown in Fig. 3a, $\Delta \boldsymbol{\psi}(\mathrm{r})$ of the armchair edge keeps increasing in the electrode region, meaning that the electrode cannot completely screen the external electric field from the Helmholtz layer at the electrode/electrolyte interface. This phenomenon is the same as the screening effect in the fewlayer graphene electrode as shown in Fig. 3c. The $\Delta \boldsymbol{\psi}(\mathrm{r})$ curve in Fig. 3a still tends to increase in the center position of the electrode, so we expect that increasing the edge plane thickness will make the $\Delta \boldsymbol{\psi}(\mathrm{r})$ continue to increase and it will plateau when the edge plane reaches a critical thickness. Thus, the armchair edge is not a promising candidate for the EDLC electrode material. Compared with armchair edges, zigzag edges show different $\Delta \boldsymbol{\psi}(\mathrm{r})$ in the electrode region: as shown in Fig. 3b, $\Delta \boldsymbol{\psi}(\mathrm{r})$ increases steeply at the edge region and then becomes flat inside the electrode. The external electric field only penetrates into a small distance in the zigzag edge and then it is completely screened. Thus, the zigzag edge has a higher capacitance than the armchair edge and the basal plane.

\subsection{Potential drop and capacitance contribution for graphene edge}

We next analyze the contributions to $C_{\text {tot }}$. Unlike the case of few-layer graphene where we can clearly distinguish three contributions (quantum, dielectric, and EDL), we cannot separate the EDL and dielectric screening parts in the potential drop for the edge planes because the electrode-electrolyte interface (dashed lines in Fig. 3a,b) is hard to define. Thus, in this work they are grouped together as a non-quantum contribution, which together with the quantum capacitance yields $\mathrm{C}_{\text {tot }}$ (Eq. 2). Fig. 3d shows that the armchair edge has a similar potential contribution to a 10-layer graphene and both contain significant contributions from quantum capacitance. The calculated areal capacitance of each electrode is listed in Table 2. The predicted total capacitance of the armchair edge is $6.12 \mu \mathrm{F} / \mathrm{cm}^{2}$, which is lower than the capacitance of the 10-layer graphene $\left(6.52 \mu \mathrm{F} / \mathrm{cm}^{2}\right)$. Compared with the armchair edge and the 10-layer graphene, the zigzag edge shows very different results: it has a lower electrostatic potential drop inside the electrode region (in Fig. 3b). In addition, the quantum contribution to the potential drop is also 
negligible due to the high $\mathrm{C}_{\mathrm{Q}}$ of zigzag edge (Table 2) and its large DOS at the Fermi level (Fig. S6). The calculated capacitance of zigzag edge is $9.04 \mu \mathrm{F} / \mathrm{cm}^{2}$, significantly higher than those of the armchair edge and the few-layer graphene.

Recent experiment showed that the edge-enriched graphene showed about $50 \%$ capacitance enhancement over that of the graphene basal plane [39], while theoretical understanding on this capacitance increase focuses on the $\mathrm{C}_{\mathrm{Q}}$ issue [42]. Our work here shows that the capacitance enhancement depends on the edge type.

\section{Table 2}

Quantum $\left(\mathrm{C}_{\mathrm{Q}}\right)$ and non-quantum $\left(\mathrm{C}_{\mathrm{NQ}}\right)$ contributions to capacitance for the graphene edge planes in comparison with the basal plane of a 10-layer graphene, according to Eq. 2.

\begin{tabular}{cccc}
\hline Electrode & $\mathrm{C}_{\text {tot }}\left(\boldsymbol{\mu F} / \mathrm{cm}^{2}\right)$ & $\mathrm{C}_{\mathrm{Q}}\left(\boldsymbol{\mu F} / \mathrm{cm}^{2}\right)$ & $\mathrm{C}_{\mathrm{NQ}}\left(\boldsymbol{\mu F} / \mathrm{cm}^{2}\right)$ \\
\hline Armchair-5 & 6.12 & 22.83 & 8.37 \\
Zigzag-5 & 9.04 & 1142 & 9.11 \\
10-layer graphene & 6.52 & 56.18 & 7.38 \\
\hline
\end{tabular}

\subsection{Classical molecular dynamics (CMD) study on the armchair and zigzag edge}

In our JDFT study of the edge planes above, we cannot separate the EDL and dielectric contributions in $\mathrm{C}_{\mathrm{NQ}}$. To examine the EDL structure and capacitance of the edge planes, we used CMD to study the armchair and zigzag edges in contact with a $6 \mathrm{M} \mathrm{NaCl}$ aqueous solution. To find out how the surface morphology would influence the EDL capacitance, we charged the surface in two different ways: (i) the surface carbon atoms were assigned with the partial charges from force fields (labeled as FF); (ii) the partial charges on the surface carbon atoms were manually set to zero (labeled as non-FF). In both cases, additional electrode charges were homogenously added to the surface $\mathrm{H}$ atoms when charging the electrodes because the JDFT study above showed that the excess charge mainly resides on the surface atoms (Fig. S2). The non-FF case can directly show how the surface charge inhomogeneity affects EDL capacitance, 
while the FF case gives the combined consequence of surface morphology and partial charges inside the electrode.

The differential EDL capacitance from CMD is plotted in Fig. 4. Because the graphene edge plane is non-flat, we need to consider the effect of solvent accessible surface area (SASA; Table 3). We plotted both SASA-normalized (Fig. 4a) and un-normalized (Fig. 4b) EDL capacitances. One can see that the edge plane always has the higher EDL capacitance than the basal plane, but the difference is smaller for the SASA-normalized one; so the SASA is one of the reasons for the higher EDL capacitance of the edge plane than the basal plane. Fig. 4a shows that near the PZC, the EDL capacitances of edge planes are about 20 to $25 \mu \mathrm{F} / \mathrm{cm}^{2}$, and decrease as the potential goes up. When there is less surface charge inhomogeneity (non-FF), the differential capacitance is smaller at low potentials. Overall, the higher EDL capacitance of the zigzag edge is in line with our JDFT results for both the total capacitance and the non-quantum contribution that contains the EDL part (Table 2).
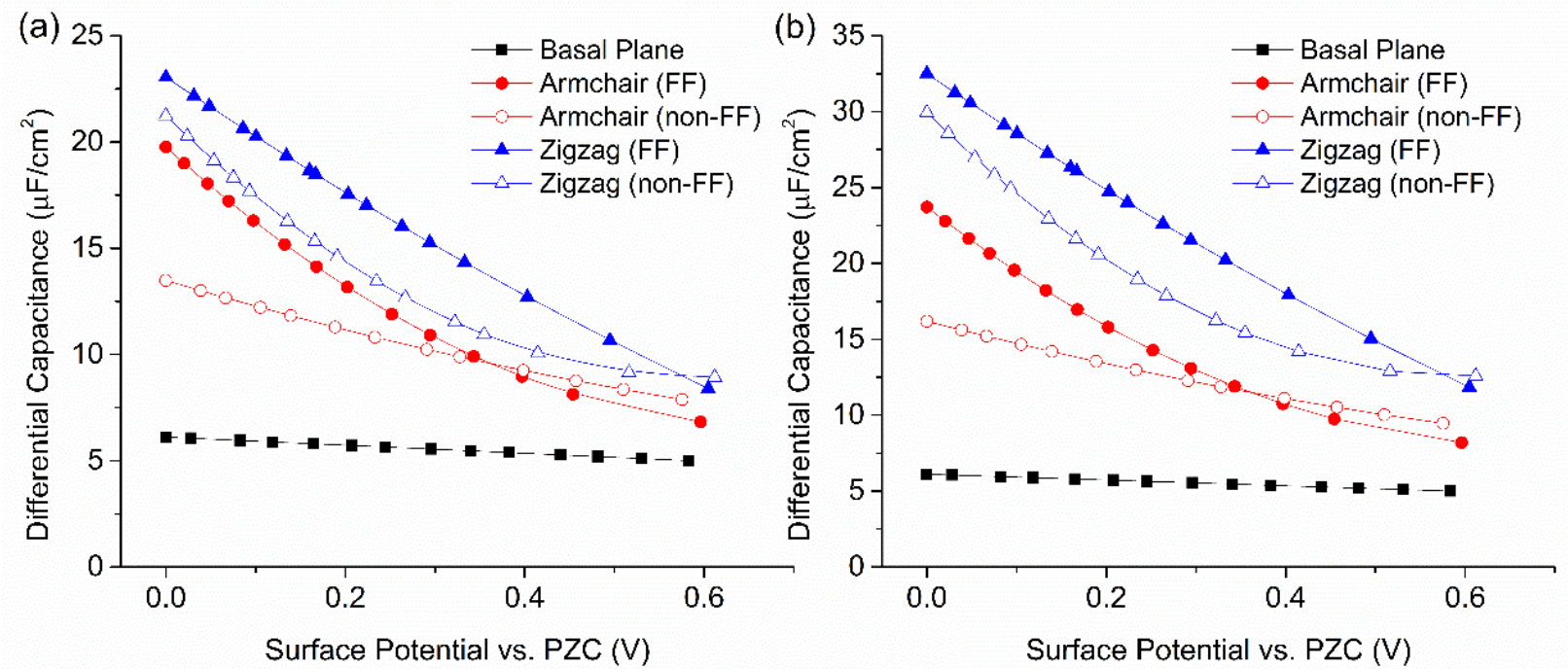

Fig. 4. Differential capacitance of graphene basal and edge planes from classical molecular dynamics simulations: (a) solvent accessible surface area (SASA)-normalized; (b) un-normalized, surface area determined by the lateral cell size. FF means the partial charges of carbon atoms are from the force field, while non-FF means that the partial charge on carbon atoms is manually set to be zero. Charging is realized by changing the charges on the surface $\mathrm{H}$ atoms. 
Table 3

Solvent accessible surface area (SASA) relative to that of the basal plane.

\begin{tabular}{cc}
\hline Electrode & Solvent Accessible Surface Area (SASA) \\
\hline Basal Plane & 1 \\
Armchair Edge & 1.2 \\
Zigzag Edge & 1.41 \\
\hline
\end{tabular}

The ion distribution and EDL structure at various potentials are plotted in Fig. 5. With the same potential drop, the responses of counter-ions are shown in Fig. 5c and 5d. One can see that the edge planes have higher counter-ion $\left(\mathrm{Cl}^{-}\right)$concentrations near the electrode surface than the basal plane, which means that the edge planes could form a thinner Helmholtz layer and hence higher EDL capacitance. Thus, there are two factors causing the EDL capacitance increase at the edge. First, the surface morphology of graphene edges favors adsorption of $\mathrm{Cl}^{-}$. The counter-ion in the FF cases also has higher peaks than in the non-FF cases, indicating that surface charge inhomogeneity also makes the EDL capacitance higher. Second, the corrugation on the edge planes leads to higher SASA which at the same bias potential can induce a stronger counter ion response, thereby yielding higher EDL capacitance.
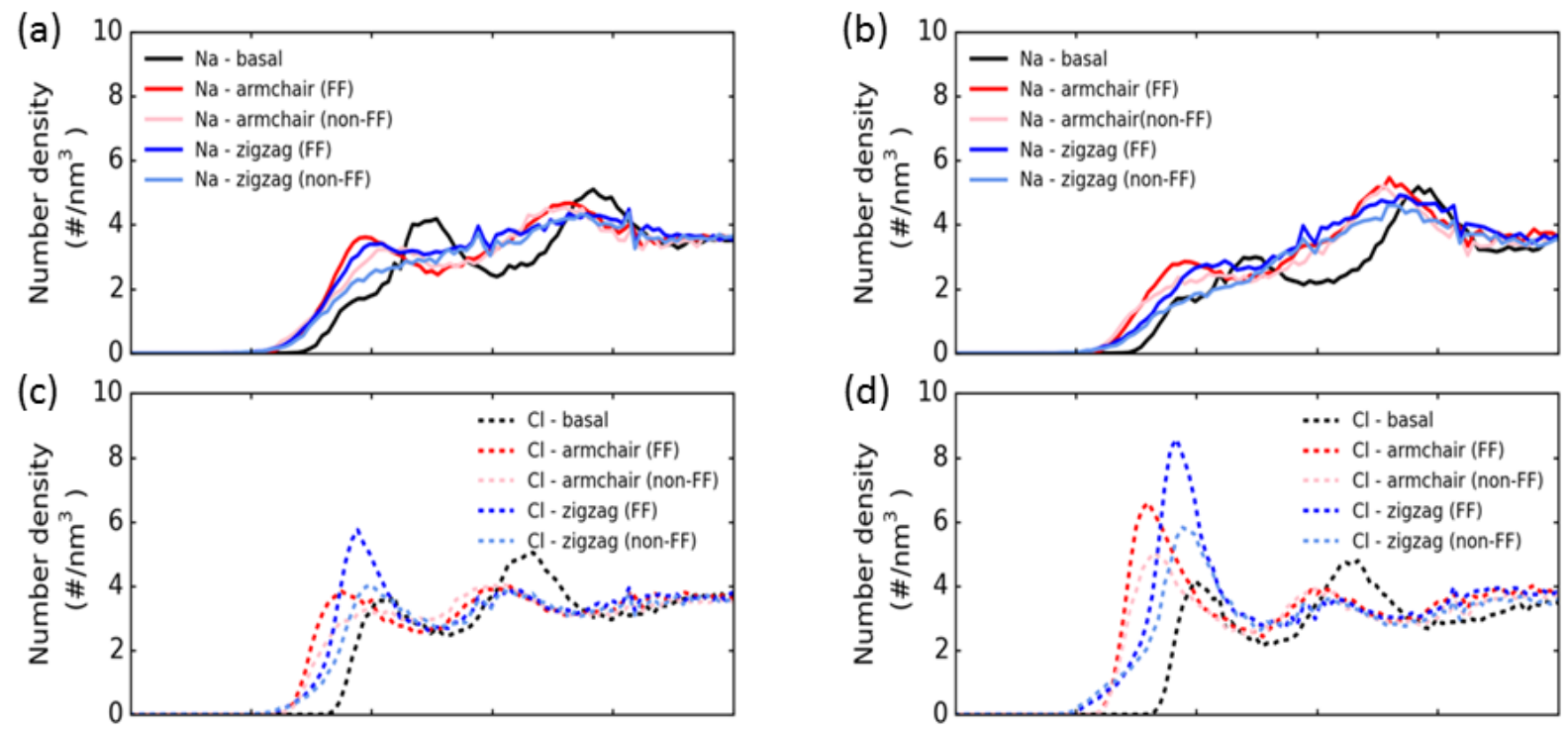

Fig. 5. $\mathrm{Na}^{+}(\mathrm{a}, \mathrm{b})$ and $\mathrm{Cl}^{-}(\mathrm{c}, \mathrm{d})$ distributions in the electrolyte at PZC (left panel) and $0.3 \mathrm{~V}$ vs PZC (right panel) from classical MD simulations for various electrodes; FF means the partial 
charges of carbon atoms are from the force field, while non-FF means that the partial charge on carbon atoms is manually set to be zero. Charging is realized by changing the charges on the surface $\mathrm{H}$ atoms.

\subsection{Combining JDFT and CMD results}

The JDFT results offer the total capacitance together with the quantum and non-quantum contributions, while the CMD simulations provide the EDL capacitance. To estimate the third contribution due to the dielectric screening, we plotted the potential drops by combining both JDFT and CMD results in Fig. 6. The dielectric contribution is separated from the non-quantum potential drop (obtained from JDFT) by subtracting the EDL contribution (obtained from CMD). The calculated dielectric capacitance is $19.28 \mu \mathrm{F} / \mathrm{cm}^{2}$ for the armchair edge and $13.33 \mu \mathrm{F} / \mathrm{cm}^{2}$ for the zigzag edge, in agreement with the different electronic structures between the armchair and zigzag edges (Fig. S5 and S6). Here again we note that due to the less-defined electrode/electrolyte boundary for the edge planes, the separation of the dielectric and EDL contributions for them can be subject to large uncertainty.

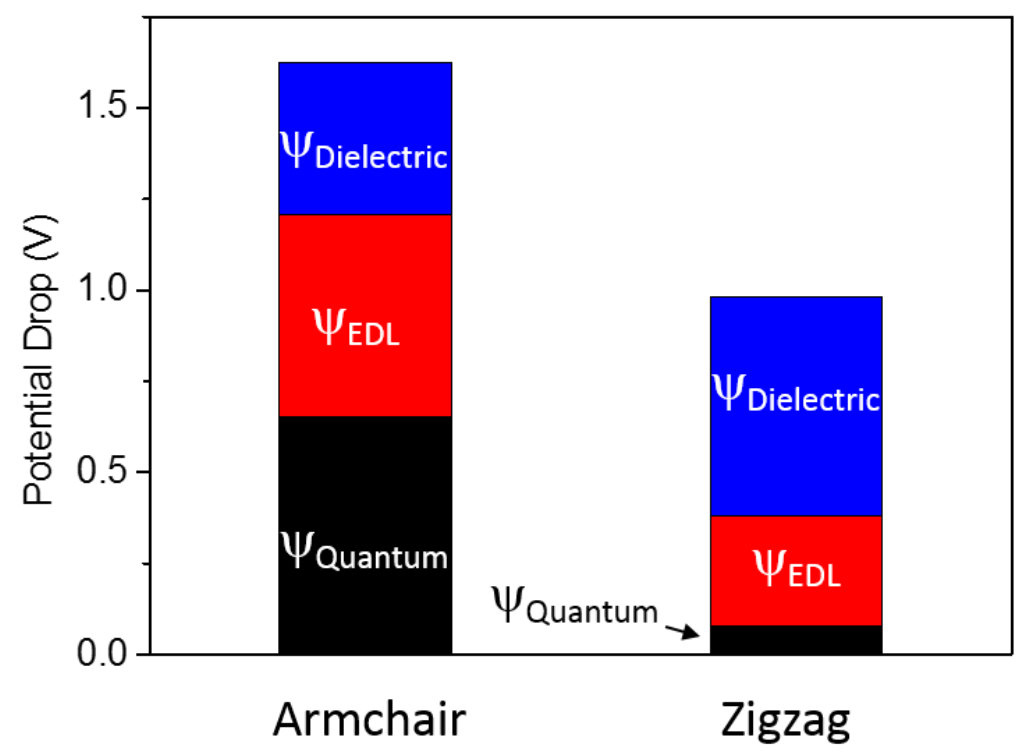

Fig. 6. Comparison of the potential drop contributions between armchair and zigzag edges by combining JDFT and CMD data. The surface charge density is $8 \mu \mathrm{C} / \mathrm{cm}^{2}$ in both cases. The 
dielectric contribution is calculated by subtracting the CMD-obtained EDL potential drop from the non-quantum contribution obtained by JDFT.

The areal capacitance of pristine graphene is about $6 \mu \mathrm{F} / \mathrm{cm}^{2}$, which has been reported by many experimental studies $[13,18,54]$. Liu et al. reported the experimentally measured areal capacitance of edge-enriched graphene nano-ribbon at about $10 \mu \mathrm{F} / \mathrm{cm}^{2}$ at low scan rates [39], which shows good consistency with our results in Table 2 for zigzag edges. Recently published experimental work by Cen et al. also found that the edge plane has higher capacitance than the basal plane [41]. More recently, Yang et al. [55] studied the EDL capacitance of carbonterminated graphene edge planes via MD simulations and found that the EDL capacitance is enhanced at the edge plane, especially for an interlayer spacing of $\sim 5.0 \AA$ A. Unlike in simple edge planes, the role of edge effect in nanoporous carbon can be very different; for example, Merlet et al. [56] studied EDL in realistic porous carbon model with MD and found that edge site (carbon electrode with concave curvature) tends to retain lower local charge than other configurations, indicating the importance of nanoconfinement.

Atomic level imaging [57] and DFT calculations [58, 59] have indicated that the zigzag edge is more favorable than the armchair edge on metal surfaces. The experimental synthesis of the pure zigzag graphene edge has been achieved by Fasel et al [60]. We think that the experimentally observed capacitance enhancement in graphene nano-ribbons may be attributed more to the zigzag edge than to the armchair edge [39].

\section{Summary and conclusions}

We have studied the capacitance of graphene edge planes in contact with an electrolyte. Joint density functional theory with an implicit solvation model showed that the zigzag edge has higher capacitance than the armchair edge and the basal plane due to the edge state (giving extremely large quantum capacitance) and the combined effect of dielectric screening and EDL response. Classical molecular dynamics (CMD) with an explicit electrolyte revealed the EDL capacitance in the order of zigzag edge > armchair edge > basal plane. The edge planes have higher solvent-accessible surface area and surface charge inhomogeneity that favors the adsorption of counter-ions, leading to higher capacitance. Our theoretical work therefore provides a complete picture of the various factors contributing to the charge capacitive 
performance of graphene edges and suggests that the zigzag graphene edge should be created for enhanced capacitance.

\section{Acknowledgement}

This research is sponsored by the Fluid Interface Reactions, Structures, and Transport (FIRST) Center, an Energy Frontier Research Center funded by the U.S. Department of Energy (DOE), Office of Science, Office of Basic Energy Sciences. This research used resources of the National Energy Research Scientific Computing Center, a DOE Office of Science User Facility supported by the Office of Science of the U.S. Department of Energy under Contract No. DE-AC02$05 \mathrm{CH} 11231$.

\section{References}

[1] M. Stengel, N. A. Spaldin, Origin of the dielectric dead layer in nanoscale capacitors, Nature 443 (7112) (2006) 679-682.

[2] V. O. Ozcelik, S. Ciraci, High-performance planar nanoscale dielectric capacitors, Phys. Rev. B 91 (19) (2015) 195445.

[3] V. O. Ozcelik, S. Ciraci, Nanoscale dielectric capacitors composed of graphene and boron nitride layers: a first-principles study of high capacitance at nanoscale, J. Phys. Chem. C 117 (29) (2013) 15327-15334.

[4] L. L. Zhang, X. S. Zhao, Carbon-based materials as supercapacitor electrodes, Chem. Soc. Rev. 38 (9) (2009) 2520-2531.

[5] P. Simon, Y. Gogotsi, B. Dunn, Where do batteries end and supercapacitors begin?, Science 343 (6176) (2014) 1210-1211. 
[6] D. R. Rolison, J. W. Long, J. C. Lytle, A. E. Fischer, C. P. Rhodes, T. M. McEvoy, M. E. Bourga, A. M. Lubers, Multifunctional 3D nanoarchitectures for energy storage and conversion, Chem. Soc. Rev. 38 (1) (2009) 226-252.

[7] P. Simon, Y. Gogotsi, Materials for electrochemical capacitors, Nat. Mater. 7 (11) (2008) 845-854.

[8] P. F. Zhang, Z. A. Qiao, Z. Y. Zhang, S. Wan, S. Dai, Mesoporous graphene-like carbon sheet: high-power supercapacitor and outstanding catalyst support, J. Mater. Chem. A 2 (31) (2014) 12262-12269.

[9] Y. P. Zhai, Y. Q. Dou, D. Y. Zhao, P. F. Fulvio, R. T. Mayes, S. Dai, Carbon materials for chemical capacitive energy storage, Adv. Mater. 23 (42) (2011) 4828-4850.

[10] Y. Wang, Z. Q. Shi, Y. Huang, Y. F. Ma, C. Y. Wang, M. M. Chen, Y. S. Chen, Supercapacitor devices based on graphene materials, J. Phys. Chem. C 113 (30) (2009) 1310313107.

[11] M. D. Stoller, S. J. Park, Y. W. Zhu, J. H. An, R. S. Ruoff, Graphene-based ultracapacitors, Nano Lett. 8 (10) (2008) 3498-3502.

[12] D. Saha, Y. C. Li, Z. H. Bi, J. H. Chen, J. K. Keum, D. K. Hensley, H. A. Grappe, H. M. Meyer, S. Dai, M. P. Paranthaman, et al., Studies on supercapacitor electrode material from activated lignin-derived mesoporous carbon, Langmuir 30 (3) (2014) 900-910.

[13] H. Ji, X. Zhao, Z. Qiao, J. Jung, Y. Zhu, Y. Lu, L. L. Zhang, A. H. MacDonald, R. S. Ruoff, Capacitance of carbon-based electrical double-layer capacitors, Nat. Commun. 5 (2014) 3317.

[14] C. H. Hou, C. D. Liang, S. Yiacoumi, S. Dai, C. Tsouris, Electrosorption capacitance of nanostructured carbon-based materials, J. Colloid Interface Sci. 302 (1) (2006) 54-61.

[15] J. Chmiola, C. Largeot, P. L. Taberna, P. Simon, Y. Gogotsi, Monolithic carbide-derived carbon films for micro-supercapacitors, Science 328 (5977) (2010) 480-483.

[16] J. L. Xia, F. Chen, J. H. Li, N. J. Tao, Measurement of the quantum capacitance of graphene, Nat. Nanotechnol. 4 (8) (2009) 505-509.

[17] L. Wang, X. Chen, W. Zhu, Y. Wang, C. Zhu, Z. Wu, Y. Han, M. Zhang, W. Li, Y. He, et al., Detection of resonant impurities in graphene by quantum capacitance measurement, Phys. Rev. B 89 (7) (2014) 075410.

[18] M. D. Stoller, C. W. Magnuson, Y. W. Zhu, S. Murali, J. W. Suk, R. Piner, R. S. Ruoff, Interfacial capacitance of single layer graphene, Energ Environ. Sci. 4 (11) (2011) 4685-4689. 
[19] P. Brooksby, A. Farquhar, H. Dykstra, M. Waterland, A. Downard, Quantum capacitance of aryldiazonium modified large area few-layer graphene electrodes, J. Phys. Chem. C 119 (46) (2015) 25778-25785.

[20] E. Paek, A. J. Pak, G. S. Hwang, On the influence of polarization effects in predicting the interfacial structure and capacitance of graphene-like electrodes in ionic liquids, J. Chem. Phys. 142 (2) (2015) 024701.

[21] E. Paek, A. J. Pak, G. S. Hwang, A computational study of the interfacial structure and capacitance of graphene in $[\mathrm{BMIM}]\left[\mathrm{PF}_{6}\right]$ ionic liquid, J. Electrochem. Soc. 160 (1) (2013) A1A10.

[22] S. N. Punnathanam, A Gibbs-ensemble based technique for Monte Carlo simulation of electric double layer capacitors (EDLC) at constant voltage, J. Chem. Phys. 140 (17) (2014)

[23] K. Kiyohara, K. Asaka, Monte carlo simulation of porous electrodes in the constant voltage ensemble, J. Phys. Chem. C 111 (43) (2007) 15903-15909.

[24] K. Kiyohara, K. Asaka, Monte carlo simulation of electrolytes in the constant voltage ensemble, J. Chem. Phys. 126 (21) (2007)

[25] L. L. Zhang, X. Zhao, H. X. Ji, M. D. Stoller, L. F. Lai, S. Murali, S. Mcdonnell, B. Cleveger, R. M. Wallace, R. S. Ruoff, Nitrogen doping of graphene and its effect on quantum Capacitance, and a new insight on the enhanced capacitance of N-doped carbon, Energ Environ. Sci. 5 (11) (2012) 9618-9625.

[26] C. Zhan, Y. Zhang, P. Cummings, D. E. Jiang, Enhancing graphene capacitance by nitrogen: effects of doping configuration and concentration, Phys. Chem. Chem. Phys. 18 (6) (2016) 46684674.

[27] C. Zhan, P. Zhang, S. Dai, J. D.E., Boron supercapacitors, ACS Energy Lett. 1 (6) (2016) 1241-1246.

[28] J. Vatamanu, X. J. Ni, F. Liu, D. Bedrov, Tailoring graphene-based electrodes from semiconducting to metallic to increase the energy density in supercapacitors, Nanotechnology 26 (46) (2015) 464001.

[29] A. J. Pak, E. Paekw, G. S. Hwang, Relative contributions of quantum and double layer capacitance to the supercapacitor performance of carbon nanotubes in an ionic liquid, Phys. Chem. Chem. Phys. 15 (45) (2013) 19741-19747. 
[30] E. Paek, A. J. Pak, K. E. Kweon, G. S. Hwang, On the origin of the enhanced supercapacitor performance of nitrogen-doped graphene, J. Phys. Chem. C 117 (11) (2013) 5610-5616.

[31] G. M. Yang, H. Z. Zhang, X. F. Fan, W. T. Zheng, Density functional theory calculations for the quantum Capacitance performance of graphene-based electrode material, J. Phys. Chem. C 119 (12) (2015) 6464-6470.

[32] E. Paek, A. J. Pak, G. S. Hwang, Curvature effects on the interfacial capacitance of carbon nanotubes in an ionic liquid, J. Phys. Chem. C 117 (45) (2013) 23539-23546.

[33] M. Mousavi-Khoshdel, E. Targholi, M. J. Momeni, First-principles calculation of quantum capacitance of codoped graphenes as supercapacitor electrodes, J. Phys. Chem. C 119 (47) (2015) 26290-26295.

[34] C. Zhan, D. Jiang, Contribution of dielectric screening to the total capacitance of few-layer graphene electrodes, J. Phys. Chem. Lett 7 (5) (2016) 789.

[35] B. C. Wood, T. Ogitsu, M. Otani, J. Biener, First-principles-inspired design strategies for graphene-based supercapacitor electrodes, J. Phys. Chem. C 118 (1) (2014) 4-15.

[36] B. Dyatkin, Y. Zhang, E. Mamontov, A. I. Kolesnikov, Y. Q. Cheng, H. M. Meyer, P. T. Cummings, Y. Gogotsi, Influence of surface oxidation on ion dynamics and capacitance in porous and nonporous carbon electrodes, J. Phys. Chem. C 120 (16) (2016) 8730-8741.

[37] W. J. Yuan, Y. Zhou, Y. R. Li, C. Li, H. L. Peng, J. Zhang, Z. F. Liu, L. M. Dai, G. Q. Shi, The edge- and basal-plane-specific electrochemistry of a single-layer graphene sheet, Sci. Rep. 3 (2013) 2248.

[38] M. A. Pope, I. A. Aksay, Four-fold increase in the intrinsic capacitance of graphene through functionalization and lattice disorder, J. Phys. Chem. C 119 (35) (2015) 20369-20378.

[39] C. Zheng, X. F. Zhou, H. L. Cao, G. H. Wang, Z. P. Liu, Edge-enriched porous graphene nanoribbons for high energy density supercapacitors, J. Mater. Chem. A 2 (20) (2014) 74847490.

[40] S. Banerjee, J. Shim, J. Rivera, X. Z. Jin, D. Estrada, V. Solovyeva, X. You, J. Pak, E. Pop, N. Aluru, et al., Electrochemistry at the edge of a single graphene layer in a nanopore, Acs Nano 7 (1) (2013) 834-843.

[41] H. C. Yang, J. Y. Yang, Z. Bo, S. Zhang, J. H. Yan, K. F. Cen, Edge effects in verticallyoriented graphene based electric double-layer capacitors, J. Power Sources 324 (2016) 309-316. 
[42] A. J. Pak, E. Paek, G. S. Hwang, Impact of graphene edges on enhancing the performance of electrochemical double layer capacitors, J. Phys. Chem. C 118 (38) (2014) 21770-21777.

[43] K. Letchworth-Weaver, T. A. Arias, Joint density functional theory of the electrodeelectrolyte interface: application to fixed electrode potentials, interfacial capacitances, and potentials of zero charge, Phys. Rev. B 86 (7) (2012) 075140.

[44] R. Sundararaman, W. A. Goddard, The Charge-Asymmetric Nonlocally Determined LocalElectric (CANDLE) solvation model, J. Chem. Phys. 142 (6) (2015) 064107.

[45] J. P. Perdew, K. Burke, M. Ernzerhof, Generalized gradient approximation made simple, Phys. Rev. Lett. 77 (18) (1996) 3865-3868.

[46] K. F. Garrity, J. W. Bennett, K. M. Rabe, D. Vanderbilt, Pseudopotentials for highthroughput DFT calculations, Comput. Mater. Sci. 81 (2014) 446-452.

[47] M. M. James, T.; Schulz, R.; Smith, J. C.; Hess, B.; Lindahl, E., GROMACS: High performance molecular simulations through multi-level parallelism from laptops to supercomputers, SoftwareX 2 (2015) 19-25.

[48] I. C. Yeh, M. L. Berkowitz, Ewald summation for systems with slab geometry, J. Chem. Phys. 111 (7) (1999) 3155-3162.

[49] T. Darden, D. York, L. Pedersen, Particle mesh ewald - an N.Log(N) method for ewald sums in large systems, J. Chem. Phys. 98 (12) (1993) 10089-10092.

[50] H. J. C. Berendsen, J. P. M. Postma, W. F. Vangunsteren, A. Dinola, J. R. Haak, Moleculardynamics with coupling to an external bath, J. Chem. Phys. 81 (8) (1984) 3684-3690.

[51] C. Merlet, C. Pean, B. Rotenberg, P. A. Madden, P. Simon, M. Salanne, Simulating supercapacitors: can we model electrodes as constant charge surfaces?, J Phys. Chem. Lett. 4 (2) (2013) 264-268.

[52] J. Poon, C. Batchelor-McAuley, K. Tschulik, R. G. Compton, Single graphene nanoplatelets: capacitance, potential of zero charge and diffusion coefficient, Chem. Sci. 6 (5) (2015) 28692876.

[53] D. G. Kvashnin, P. B. Sorokin, J. W. Bruning, L. A. Chernozatonskii, The impact of edges and dopants on the work function of graphene nanostructures: the way to high electronic emission from pure carbon medium, Appl. Phys. Lett. 102 (18) (2013) 183112.

[54] E. Uesugi, H. Goto, R. Eguchi, A. Fujiwara, Y. Kubozono, Electric double-layer capacitance between an ionic liquid and few-layer graphene, Sci. Rep. 3 (2013) 1595. 
[55] H. Yang, X. Zhang, J. Yang, Z. Bo, M. Hu, J. Yan, K. F. Cen, Molecular origin of electric double-layer capacitance at multilayer graphene edges, J. Phys. Chem. Lett. 8 (1) (2017) 153160.

[56] C. Merlet, C. Pean, B. Rotenberg, P. A. Madden, B. Daffos, P. L. Taberna, P. Simon, M. Salanne, Highly confined ions store charge more efficiently in supercapacitors, Nat. Commun. 4 (2013) 2701.

[57] J. F. Tian, H. L. Cao, W. Wu, Q. K. Yu, Y. P. Chen, Direct imaging of graphene edges: atomic structure and electronic scattering, Nano Lett. 11 (9) (2011) 3663-3668.

[58] H. B. Shu, X. S. Chen, X. M. Tao, F. Ding, Edge structural stability and kinetics of graphene chemical vapor deposition growth, Acs Nano 6 (4) (2012) 3243-3250.

[59] D. S. Wei, F. Wang, Relative stability of armchair, zigzag and reczag graphene edges on the $\mathrm{Ru}(0001)$ surface, Surf. Sci. 606 (3-4) (2012) 485-489.

[60] P. Ruffieux, S. Y. Wang, B. Yang, C. Sanchez-Sanchez, J. Liu, T. Dienel, L. Talirz, P. Shinde, C. A. Pignedoli, D. Passerone, et al., On-surface synthesis of graphene nanoribbons with zigzag edge topology, Nature 531 (7595) (2016) 489-492. 
${ }^{\star}$ Graphical Abstract

ToC figure

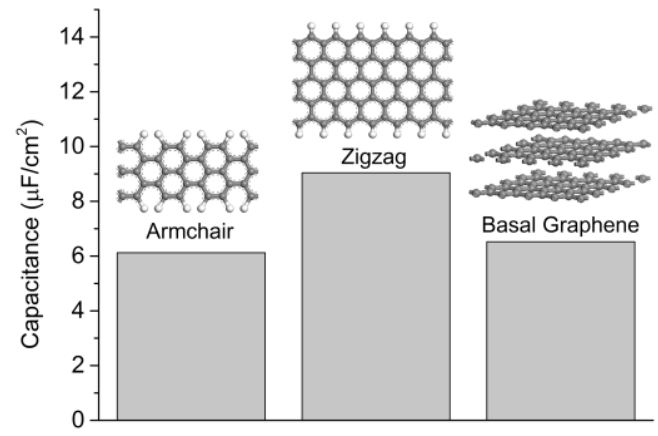

